\title{
Telemedicine Versus Face-to-Face Evaluations by Respiratory Therapists of Mechanically Ventilated Neonates and Children: A Pilot Study
}

\author{
Rebecca C Bell MD MPH, Phoebe H Yager MD, Maureen E Clark MSc, \\ Serguei Roumiantsev MD PhD, Heather L Venancio RRT, Daniel W Chipman RRT, \\ Robert M Kacmarek PhD RRT FAARC, and Natan N Noviski MD
}

\begin{abstract}
BACKGROUND: Mechanical ventilation is one of the most important therapeutic interventions in neonatal and pediatric ICUs. Telemedicine has been shown to reliably extend pediatric intensivist expertise to facilities where expertise is limited. If reliable, telemedicine may extend the reach of pediatric respiratory therapists (RTs) to facilities where expertise does not exist or free up existing RT resources for important face-to-face activities in facilities where expertise is limited. The aim of this study was to determine how well respiratory assessments for ventilated neonates and children correlated when performed simultaneously by 2 RTs face-to-face and via telemedicine. METHODS: We conducted a pilot study including 40 assessments by 16 RTs on 11 subjects (5 neonatal ICU; 6 pediatric ICU). Anonymously completed intake forms by 2 different RTs concurrently assessing 14 ventilator-derived and patient-based respiratory variables were used to determine correlations. RESULTS: Forty paired assessments were performed. Median telemedicine assessment time was $8 \mathrm{~min}$. The Pearson correlation coefficient ( $r$ ) was used to determine agreement between continuous data, and the Cohen kappa statistics were used for binary variables. Pressure control, PEEP, breathing frequency, and $\mathrm{F}_{\mathrm{IO}_{2}}$ perfectly correlated $(\mathrm{r}=1$, all $P<.001)$ as did the presence of a $\mathrm{CO}_{2}$ monitor and need for increased ventilatory support $($ kappa $=1)$. The Pearson correlation coefficient for $\mathrm{V}_{\mathrm{T}}$, minute ventilation, mean airway pressure, and oxygen saturation ranged from 0.84 to 0.97 (all $P<.001)$. kappa $=0.41(95 \%$ CI $0.02-0.80)$ for patient-triggered breaths, and kappa $=0.57$ (95\% CI 0.19-0.94) for breathing frequency higher than set frequency. kappa $=-0.25(95 \%$ CI -0.46 to -0.04$)$ for need for suctioning. CONCLUSIONS: Telemedicine technology was acceptable to RTs. Telemedicine evaluations highly correlated with face-to-face for 10 of 14 aspects of standard bedside respiratory assessment. Poor correlation was noted for more complex, patientgenerated parameters, highlighting the importance of further investigation incorporating a virtual stethoscope. Key words: telemedicine; pediatrics; intensive care; physical examination; reliability of results. [Respir Care 2016;61(2):149-154. (C) 2016 Daedalus Enterprises]
\end{abstract}

\section{Introduction}

Mechanical ventilation is one of the most important therapeutic interventions provided in pediatric and neona-

\footnotetext{
Dr Bell is affiliated with the Division of Inpatient and Critical Care Pediatrics, University of Vermont Children's Hospital, Burlington, Vermont. Dr Yager, Ms Clark, and Dr Noviski are affiliated with the Division of Pediatric Critical Care, Department of Pediatrics; Dr Roumiantsev is affiliated with the Division of Neonatology and Newborn Medicine, Department of Pediatrics; and Ms Venancio, Mr Chipman, and
}

tal ICUs. However, its use is difficult and demanding, and the approach can involve high risk of serious complica-

\footnotetext{
Dr Kacmarek are affiliated with the Department of Respiratory Care, Massachusetts General Hospital, Boston, Massachusetts.

This work was supported by the Department of Pediatrics, Massachusetts General Hospital, Boston, Massachusetts. Dr Kacmarek consults for Orange Medical and has received research grants from Covidien and Venner Medical. Drs Bell, Yager, Roumiantsev, Noviski, Ms Clark, Ms Venancio and Mr Chipman have disclosed no conflicts of interest.
} 
tions. ${ }^{1}$ Telemedicine can improve deployment of critical care resources ${ }^{2}$ and provide a way to extend the reach of mechanical intervention for neonates and children in remote locations. The use of telemedicine in ICUs has had a favorable impact on both patient care and health-care organizations. ${ }^{3}$ Its use has expanded in scope and practice over the past decade, especially in large United States tertiary centers. ${ }^{4}$

Several studies have demonstrated significant mortality reductions and cost savings associated with the use of telemedicine technology for remote, continuous monitoring of adult ICU subjects by critical care specialists. ${ }^{5,6}$ Between 2003 and 2010, the number of hospitals in the United States using ICU telemedicine rose from $16(0.4 \%$ of total) to 213 (4.6\% of total); the number of ICU beds covered by telemedicine increased from 598 ( $0.9 \%$ of total) to 5,799 (7.9\% of total). ${ }^{4}$ At last count, an estimated $10 \%$ of ICU beds in the United States were covered by ICU telemedicine in the form of continuous remote monitoring, with many more using some other type of remote care. $^{7}$

Pediatric-specific uses for telemedicine in the ICU are also vast and include (1) urgent telemedicine consultations with pediatric intensivists, neonatologists, and cardiac intensivists for children in underserved rural emergency departments or ICUs lacking such expertise ${ }^{8-12}$; (2) teleconsultations with pediatric subspecialists in infectious diseases, cardiology, and neurology for children in community hospitals without local access to these subspecialists ${ }^{13}$; and (3) nighttime telecommunication between attending pediatric intensivists on home-call with fellows and residents caring for pediatric ICU patients in a teaching hospital to enhance continuity of care and augment team communication during off hours. ${ }^{14}$

Although a wide body of research exists to explore how best to utilize telemedicine to extend the reach of a limited cadre of critical care subspecialty physicians, ${ }^{15}$ little has been done to investigate how this technology can be used by non-physician specialists, including respiratory therapists (RTs), for whom the scope of practice is rapidly increasing. ${ }^{16-19}$ At this time, there are no published studies on the roles of RTs in ICU telemedicine. Similarly, there is no research on the use of telemedicine in respiratory

\footnotetext{
Drs Bell and Yager presented a version of this paper as a poster presentation at the 12th European Conference on Pediatric and Neonatal Ventilation, held May 21-24, 2014, in Montreux, Switzerland.
}

Drs Bell and Yager are co-first authors.

Correspondence: Natan Noviski MD, 175 Cambridge Street, CPZS-5, Boston, MA 02114. E-mail: nnoviski@partners.org.

DOI: $10.4187 /$ respcare 04080

\section{QUICK LOOK}

\section{Current knowledge}

Telemedicine has achieved rapid acceptance and growth. However, there are no published studies on the role of respiratory therapists (RTs) in ICU telemedicine. Similarly, there is no research on the use of telemedicine in standard respiratory assessments of mechanically ventilated neonatal and pediatric ICU patients.

\section{What this paper contributes to our knowledge}

This study shows that RTs can reliably use telemedicine to assess set and easily observable parameters for mechanically ventilated neonatal and pediatric ICU subjects. It suggests a larger role for the use of telemedicine by RTs, but further research is needed to determine the nature and extent of that role.

assessments of mechanically ventilated neonates and children. The aim of this study was to determine whether aspects of the standard bedside respiratory evaluation performed by RTs on mechanically ventilated neonates and children could be obtained reliably via telemedicine compared with face-to-face evaluation.

\section{Methods}

The study was conducted at Massachusetts General Hospital in Boston, Massachusetts, in accordance with the principles outlined in the Declaration of Helsinki. The hospital institutional review board approved the study protocol, and written informed consent was obtained from the parents or guardians of all study subjects.

Each RT provider received an information sheet on the objectives of the research and its risks and benefits. Willingness to perform the study assessment and complete the data collection form freely and anonymously were considered implied consent as stipulated by the Partners Human Research Committee.

\section{Study Subjects and Setting}

All mechanically ventilated neonates and children admitted to our 18-bed neonatal ICU and 14-bed pediatric ICU were eligible for the study unless they met exclusion criteria. These included age $<24 \mathrm{~h}$ or $\geq 18 \mathrm{y}$; inability to obtain parental or guardian consent; concurrent participation in another study; patient being deemed medically unstable by his or her attending physician; participation causing an unacceptable interruption in care; or patient at end of life. Mobile videoconferencing units, housed in the pe- 
diatric and neonatal ICUs, were taken into each subject's room to perform assessments. Selection of study subjects was based on the ability to contact guardians to obtain consent and represented a convenience sample. Patients $<24 \mathrm{~h}$ old were excluded at the request of neonatal ICU staff concerned that study participation could add an additional layer of stress to new parents dealing with a child just admitted to the unit.

\section{Protocol and Measurements}

Telemedicine is achieved by having a professionally integrated mobile telemedicine cart available in the pediatric/neonatal ICU and office-based units for remote evaluation by participating RTs. The connection between the mobile telemedicine cart at the patient's bedside and a remote, office-based unit uses HIPAA-compliant videoconferencing connectivity, including mechanisms to authenticate, encrypt, and decrypt electronic protected health information. The study coordinator facilitated the connection between the mobile telemedicine cart and the remote unit by rolling the mobile unit to each study subject's bedside, connecting the unit to the ethernet via wire cable, and establishing connectivity with the remote site. When not being employed for study purposes, the mobile telemedicine cart was placed in a storage area where no electronic protected health information resides. Advanced Encryption Standard 128-bit encryption secures the audio/video connection, end to end, securely traversing the firewall between the hospital's business network and the remote office unit. Additionally, the video infrastructure is secured by secure socket layer encryption at our hosting facility. This hosting facility has undergone a third party audit and obtained an SSAE 16 SOC type 2 standing. Interfaces were not recorded, thereby obviating the need for protection of stored electronic protected health information from improper alteration or destruction. Connections were made through the H.323 gateway on a Vidyo infrastructure, achieving a maximum transmission speed of 1.2 megabits/s. The video protocol being utilized on the office-based system is H.264 scalable video codec. The number of frames transmitted per second depends on the live bandwidth of the call and ranges between 20 and $30 \mathrm{frames} / \mathrm{s}$. The mobile telemedicine cart consists of a Tandberg/Cisco Intern Cart system (approximately $\$ 35,000)$ that includes a high-definition, moveable video camera, an LCD screen for viewing the remote RT, audio input/output for dialog, and accessories for close-up patient examinations (high-definition camera with $7 \times$ zoom). The office-based system employs a desktop or laptop computer loaded with Vidyo software version 2.2 (Hackensack, New Jersey) as well as a Logitech camera (approximately \$100). Vidyo conferencing software was downloaded from the internet for free. The software en- abled the user to remotely manipulate the far-end mobile telemedicine cart camera (up/down, right/left, zoom in/ out). The cost of establishing a complete Vidyo infrastructure that is H.323-capable and provides secure web-based videoconferencing ranges between $\$ 100,000$ and $\$ 500,000$. Feasibility of use of this system has been described previously by Yager et al. ${ }^{14}$

RTs with subspecialty experience caring for ventilated neonates and children in the ICU were exclusively approached to participate in the study. To ensure confidentiality and avoid the potential for coercion to participate, no data were collected regarding age, sex, or years of experience. RTs were not facile with the telemedicine equipment and were oriented to its proper use immediately before participation. For each encounter, a study coordinator was present at the subject's bedside to facilitate connectivity between the mobile telemedicine cart and the remote site. The RT performing the face-to-face assessment was instructed to assist the telemedicine RT with the remote assessment by doing things such as exposing the subject's chest or scrolling through different ventilator-based waveforms if directed to do so by the remote RT.

Two RTs not actively providing care for the study subjects were randomly selected from the available pool of RTs assigned to pediatrics on any given day. They were then randomly assigned to perform either face-to-face or telemedicine examinations. RTs assigned to cover study subjects were excluded. For a given encounter, both the face-to-face RT and the telemedicine RT were asked to perform concurrent evaluations on the same subject and to complete in real time a written data collection form including 14 aspects of the typical RT assessment performed on ventilated patients. The RT performing the telemedicine examination did so from an office outside the ICU. Ventilator-derived parameters included pressure control, PEEP, mean airway pressure, breathing frequency, $\mathrm{F}_{\mathrm{IO}_{2}}$, and inspiratory to expiratory time ratio (I-E ratio). Patientderived parameters included tidal volume $\left(\mathrm{V}_{\mathrm{T}}\right)$, minute ventilation, oxygen saturation, presence of patient-triggered breaths, and the need for suctioning or increased ventilator support. RTs were instructed to make these last 2 determinations based on available ventilator waveforms, pressure readings, and $\mathrm{V}_{\mathrm{T}}$. No remote auscultation device was available. All subjects were ventilated with SERVO-i machines (Maquet, Rastatt, Germany).

Paired RTs recorded findings for each subject anonymously on face-to-face and telemedicine intake forms that were later compared for correlations. Subject demographic variables, including age, sex, ethnicity, and weight, were recorded by the study coordinator as were subject characteristics at the time of the encounter, including whether the subject had a cuffed endotracheal tube, whether the subject 
was muscle-relaxed, and whether the subject was in an incubator.

\section{Statistical Analysis}

The Cohen kappa statistic was used to determine agreement of binary variables (eg, presence vs absence of patient-triggered breaths). The Pearson correlation coefficient (r) with a 2-tailed $P$ value and 95\% CIs was used to determine associations between continuous data $\left(\mathrm{eg}, \mathrm{V}_{\mathrm{T}}\right.$ and oxygen saturation). For the purposes of this study, face-to-face measurements were considered the accepted standard.

\section{Results}

Sixteen RTs performed 40 assessments (20 face-toface and 20 telemedicine) on 11 subjects, 6 in the pediatric ICU and 5 in the neonatal ICU. The median assessment time was $8 \mathrm{~min}$ (range 6-20 min). Telemedicine RTs reported 3 instances of technical difficulties, leading to temporary video freezing and delayed far-end camera response to remote commands to zoom in/out or move up/down and right/left. Although these difficulties caused slight delays in telemedicine assessment, they did not prevent completion of the telemedicine evaluation or prolong it by $>2$ min relative to face-to-face assessment. When RTs were asked what additional parameters they would have liked to assess, the most common answer was auscultation exams; others were endotracheal tube cuff pressure and ventilator alarm reviews.

Table 1 provides subject demographic data and a breakdown of subject diagnoses. The median age was 3 months, and the most frequent reason for intubation was respiratory failure secondary to prematurity, congenital airway anomaly, or lower respiratory infection. In $30 \%$ of encounters, subjects were nasally intubated; in $55 \%$, they had cuffed endotracheal tubes. In $85 \%$ of examinations, subjects were sedated; in $45 \%$, they were receiving neuromuscular blocking agents. One subject was on venovenous extracorporeal life support.

Correlation between raters for the values of set pressure control, PEEP, breathing frequency, and $\mathrm{F}_{\mathrm{IO}_{2}}$ was high ( $\mathrm{r}=1$ for all variables), whereas it was 0.47 for I-E ratio. The more dynamic variables of $\mathrm{V}_{\mathrm{T}}$, minute ventilation, mean airway pressure, and oxygen saturation had $\mathrm{r}$ values ranging from 0.84 to 0.97 . Table 2 shows correlations for continuous variables.

For binary data, agreement was perfect among raters on the need for an increase in ventilatory support and the presence of an end-tidal $\mathrm{CO}_{2}$ monitor (kappa $=1$ ). Correlation on the presence of patient-triggered breaths was kappa $=0.41$, and for breathing frequency being higher
Table 1. Subject Demographic Data

\begin{tabular}{lc}
\hline \hline \multicolumn{1}{c}{ Subject Characteristics } & All Subjects $(N=11)$ \\
\hline Age, median (range) & 3 months (2 weeks to 5 years) \\
Male sex, $n(\%)$ & $5(45)$ \\
Ethnicity, $n(\%)$ & \\
Non-Hispanic & $6(55)$ \\
Hispanic & $4(36)$ \\
Haitian & $1(9)$ \\
ICU, $n(\%)$ & \\
Neonatal ICU & $5(45)$ \\
Pediatric ICU & $6(55)$ \\
Weight, median (range) kg & $5(0.9-22)$ \\
Diagnosis, $n$ (\%) & \\
Respiratory distress due to prematurity & $3(27)$ \\
Congenital airway anomaly & $3(27)$ \\
Lower respiratory infection & $2(18)$ \\
Upper respiratory infection & $1(9)$ \\
Cardiac disease & $1(9)$ \\
Neurologic disease & $1(9)$ \\
Reason for intubation, $n(\%)$ & \\
Respiratory failure & $6(55)$ \\
Upper-airway obstruction & $2(18)$ \\
Status post-airway reconstruction & $2(18)$ \\
Airway protection & $1(9)$ \\
Total days of intubation, median (range) & $10(4-59)$ \\
\hline &
\end{tabular}

Table 2. Correlation of Face-to-Face and Telemedicine Ventilator and Monitor Values

\begin{tabular}{llc}
\hline \multicolumn{1}{c}{ Values } & $\mathrm{r}$ & $P$ \\
\hline Set pressure & 1 & $<.001$ \\
Set PEEP & 1 & $<.001$ \\
Set frequency & 1 & $<.001$ \\
Set $\mathrm{F}_{\mathrm{IO}_{2}}$ & 1 & $<.001$ \\
$\mathrm{I}-\mathrm{E}$ ratio & 0.47 & .040 \\
$\mathrm{~V}_{\mathrm{T}}$ & 0.84 & $<.001$ \\
Minute ventilation & 0.97 & $<.001$ \\
Mean airway pressure & 0.95 & $<.001$ \\
Oxygen saturation & 0.97 & $<.001$ \\
& & \\
\hline I-E = inspiratory-expiratory & & \\
\hline
\end{tabular}

than the set ventilator rate, it was kappa $=0.57$. The correlation on the need for suctioning was kappa $=-0.25$. Outcomes for respiratory assessment questions are shown in Table 3.

\section{Discussion}

To our knowledge, this is the first study examining the use of telemedicine by RTs to evaluate mechanically ventilated neonates and children. ${ }^{20,21}$ Results of our study indicate high correlations for 10 of 14 variables evaluated by telemedicine and face-to-face examinations. These included all ventilatorderived values except for the I-E ratio. The 3 encounters in 
Table 3. Face-to-Face and Telemedicine Agreement of Respiratory Assessment Questions

\begin{tabular}{lc}
\hline \hline \multicolumn{1}{c}{ Question } & kappa (reference range) \\
\hline Presence of patient-triggered breaths & $0.41(0.02-0.80)$ \\
Breathing frequency higher than set frequency & $0.57(0.19-0.94)$ \\
Presence of end-tidal $\mathrm{CO}_{2}$ monitor & $1.0(1.0-1.0)$ \\
Need for suctioning & $-0.25(-0.46$ to -0.04$)$ \\
Need for increased ventilator support & $1.0(1.0-1.0)$ \\
\hline
\end{tabular}

which technological difficulties prevented true simultaneous evaluation by the face-to-face and telemedicine RTs contributed to discrepancies in more dynamic aspects of the exam, including I-E ratio, $\mathrm{V}_{\mathrm{T}}$, presence of patient-triggered breaths, and breathing frequency higher than set frequency. Finally, whereas paired data indicated high agreement on the need for increased ventilator support, it is possible that some of these subjects simply needed to be suctioned. Given the poor agreement on whether a given subject needed suctioning, caution should be used in concluding that telemedicine without a remote stethoscope can reliably be used to assess the need for increased ventilator support.

Despite the cost and complexity of telemedicine ICU implementation, ${ }^{22}$ remote ICU coverage is rapidly being adopted to increase access to a dwindling supply of intensivist physicians. ${ }^{23}$ In the ICU setting, the ability to provide high-quality care at reduced cost will largely depend upon maximizing interdisciplinary care teams, which include respiratory therapists, offering expanded roles and responsibilities (recently identified by the American Association for Respiratory Care). ${ }^{19}$ Our study indicates that telemedicine can enable care equivalent to that provided by a face-to-face RT for certain activities, potentially freeing up in-house RTs to perform other less telemedicine-amenable activities and increasing the efficiency with which the RT workforce is utilized. In our model, remote RT assessment did require a second person physically in the unit to roll the mobile telemedicine cart to each subject's bedside and establish connectivity. It also relied upon the bedside RT to help with subject positioning and scrolling through ventilator screens. Whether a unit-based provider, such as a bedside nurse, would be able to serve in this role may impact the ultimate efficiency and cost savings of implementing such a program.

RTs at Massachusetts General Hospital, for example, are trained in neonatal and pediatric ventilator management. They make frequent routine and emergency consultations at the bedsides of patients admitted to the pediatric and neonatal ICUs. Many hospitals where critically ill pediatric patients are cared for in adult ICUs may not have RTs with adequate training in neonatal and pediatric respiratory care. ${ }^{24}$ Although these RTs may be facile at tube taping, checking cuff pressures, and performing bedside auscultation, they may benefit from consultation with a pediatric subspecialty-trained RT via telemedicine. In countries outside the United States where RTs either do not exist or have only recently been introduced as a new profession within the last $15 \mathrm{y}$ (eg, Germany), frequently there are no bedside RTs, and physicians and nurses are fully responsible for managing ventilators. ${ }^{25}$ In these areas, telemedicine could be used to enable RTs to remotely consult on challenging ventilated neonatal and pediatrics patients. At an urban, academic telemedicine referral center serving rural North India, Singh et $\mathrm{al}^{26}$ found that of 306 pediatric patient referrals received between 2005 and 2008, 26\% were transported into the city for further subspecialty care. Sixteen percent were deemed too unstable for transport, and videoconferencing was used to provide ongoing advice and support to the regional care providers. ${ }^{26}$ Telemedicine could improve patient outcomes when integrated into transport practices. Similarly, mechanically ventilated neonates and children in such settings may benefit from telemedicine consultations with remote physicians and RTs with pediatric critical care experience.

Our study had several limitations. The absence of remote auscultation equipment probably contributed to the weak correlation between face-to-face and telemedicine assessments of the need for subject suctioning. Review of the paired data revealed discrepancies in I-E ratio between pairs where one rater recorded the inspiratory time and the other recorded the I-E ratio. Poor instructions to RTs on how to record the I-E ratio contributed to poor correlation on this aspect of the evaluation. Technological difficulties leading to delayed video streaming to the remote RT prevented concurrent face-to-face and telemedicine exams in some instances and made it difficult to draw definitive conclusions around dynamic aspects of the assessment. Although the 14 parameters chosen for comparison in this study were based on the standard bedside respiratory evaluation performed by RTs at our institution, they did not include specific parameters requiring more interpretation, such as pressure-volume loops, chest rise, or ventilator synchrony. Although the evaluation did ask the RTs to make a determination regarding the need for increased ventilator support based on available waveforms, pressure readings, and $\mathrm{V}_{\mathrm{T}}$ levels, it did not specifically ask them to assess readiness to wean ventilator support. In addition, this single-center pilot study had a small, heterogeneous sample size and few RT-subject encounters. Lack of variability in the data and possible agreement based on chance prevented analyses of 3 assessment components: the presence of auto-PEEP, patient-ventilator asynchrony, and condensation in the ventilator tubing. 


\section{Telemedicine vs Face-To-Face Evaluations}

\section{Conclusions}

In summary, this study shows that telemedicine can be used to reliably assess easily visualized, pre-programmed parameters for mechanically ventilated patients in the pediatric and neonatal ICUs. It remains less clear whether dynamic, patient-triggered parameters can be reliably evaluated via telemedicine. Further research incorporating a virtual stethoscope and more parameters requiring RT interpretation are needed to determine the optimal use of telemedicine by RTs caring for mechanically ventilated neonates and children.

\section{REFERENCES}

1. Zielińska M, Zieliński S, Sniatkowska-Bartkowska A. Mechanical ventilation in children: problems and issues. Adv Clin Exp Med 2014;23(5):843-848.

2. Khunlertkit A, Carayon P. Contributions of tele-intensive care unit (Tele-ICU) technology to quality of care and patient safety. J Crit Care 2013;28(3):315.e1-315.e12.

3. Kumar S, Merchant S, Reynolds R. Tele-ICU: efficacy and costeffectiveness of remotely managing critical care. Perspect Health Inf Manag 2013;10(Spring):1f.

4. Kahn JM, Cicero BD, Wallace DJ, Iwashyna TJ. Adoption of ICU telemedicine in the United States. Crit Care Med 2014;42(2):362368.

5. Wilcox ME, Adhikari NK. The effect of telemedicine in critically ill patients: systematic review and meta-analysis. Crit Care 2012;16(4): R127.

6. Breslow MJ, Rosenfeld BA, Doerfler M, Burke G, Yates G, Stone DJ, et al. Effect of a multiple-site intensive care unit telemedicine program on clinical and economic outcomes: an alternative paradigm for intensivist staffing. Crit Care Med 2004;32(1):31-38.

7. Vespa PM, Miller C, Hu X, Nenov V, Buxey F, Martin NA. Intensive care unit robotic telepresence facilitates rapid physician response to unstable patients and decreased cost in neurointensive care. Surg Neurol 2007;67(4):331-337.

8. Heath B, Salerno R, Hopkins A, Hertzig J, Caputo M. Pediatric critical care telemedicine in rural underserved emergency departments. Pediatr Crit Care Med 2009;10(5):588-591.

9. Ciomartan T. Telemedicine: a useful adjunct in the care of critically ill children. Crit Care Med 2012;40(9):2731-2732.

10. Marcin JP, Schepps DE, Page KA, Struve SN, Nagrampa E, Dimand RJ. The use of telemedicine to provide pediatric critical care consultations to pediatric trauma patients admitted to a remote trauma intensive care unit: A preliminary report. Pediatr Crit Care Med 2004;5(3):251-256

11. McCoy M, Makkar A, Foulks A, Legako E. Establishing level II neonatal services in southwestern Oklahoma. J Okla State Med Assoc 2014;107(9-10):493-496.

12. Munoz RA, Burbano NH, Motoa MV, Santiago G, Klevemann M, Casilli J. Telemedicine in Pediatric Cardiac Critical Care. Telemed J E Health 2012;18(2):132-136.

13. McSwain SD, Marcin JP. Telemedicine for the care of children in the hospital setting. Pediatr Ann 2014;43(2):e44-e49.

14. Yager PH, Cummings BM, Whalen MJ, Noviski N. Nighttime telecommunication between remote staff intensivists and bedside personnel in a pediatric intensive care unit: a retrospective study. Crit Care Med 2012;40(9):2700-2703.

15. Kahn JM, Hill NS, Lilly CM Angus DC, Jacobi J, Rubenfeld GD, et al. The research agenda in ICU telemedicine: a statement from the Critical Care Societies Collaborative. Chest 2011;140(1):230-238.

16. Smith GA. Respiratory care: evolution of a profession. Lenexa, Kansas: Applied Measurement Professionals; 1989.

17. Kacmarek RM, Durbin CG, Barnes TA, Kageler WV, Walton JR, O'Neil EH. Creating a vision for respiratory care in 2015 and beyond. Respir Care 2009;54(3):375-389.

18. Commission on Accreditation of Allied Health Programs. Standards and guidelines for the profession of respiratory care, 2003. http://www.caahep.org/documents/ForProgramDirectors/ RT_Standards.pdf. Accessed January 24, 2015.

19. Barnes TA, Kacmarek RM, Kageler WV, Morris MJ. Transitioning the respiratory therapy workforce for 2015 and beyond. Respir Care 2011;56(5):681-690.

20. Epstein D, Brill JE. A history of pediatric critical care medicine. Pediatr Res 2005;58(5):987-996.

21. McConnochie KM, Conners GP, Brayer AF, Goepp J, Herendeen NE, Wood NE, et al. Differences in diagnosis and treatment using telemedicine versus in-person evaluation of acute illness. Ambul Pediatr 2006;6(4):187-195; discussion 196-187.

22. Moeckli J, Cram P, Cunningham C, Reisinger HS. Staff acceptance of a telemedicine intensive care unit program: a qualitative study. J Crit Care 2013;28(6):890-901.

23. Young LB, Chan PS, Lu X, Nallamothu BK, Sasson C, Cram PM. Impact of telemedicine intensive care unit coverage on patient outcomes: a systematic review and meta-analysis. Arch Intern Med 2011;171(6):498-506.

24. Walsh BK, Gentile MA, Grenier BM. Orienting new respiratory therapists into the neonatal/pediatric environment: A survey of educators and managers. Respir Care 2011;56(8):1122-1129.

25. Karg O, Bubulj C, Esche B, Geiseler J, Bonnet R, Mäder I. The respiratory therapist. Pneumologie 2008;62(11):685-689.

26. Singh M, Das RR. Utility of telemedicine for children in India. Indian J Pediatr 2010;77(1):73-75.

This article is approved for Continuing Respiratory Care Education credit. For information and to obtain your CRCE

(free to AARC members) visit www.rcjournal.com

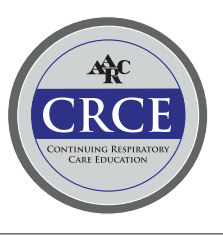

